\title{
Topology of Electronic Charge Density and Energetics of Planar Faults in fec Metals
}

\author{
Nicholas Kioussis, M. Herbranson, and E. Collins \\ Department of Physics, California State University Northridge, Northridge, California 91330-8268 \\ M.E. Eberhart \\ Department of Chemistry and Geochemistry, Colorado School of Mines, Golden, Colorado 80401
}

(Received 29 October 2001; published 6 March 2002)

\begin{abstract}
Using $a b$ initio calculations we have studied the energetics and the evolution of the electronic charge density with shear in three fcc metals exhibiting different deformation properties, aluminum, silver, and iridium. The charge redistribution described by the change in character of specific charge density critical points (cps), is ascertained from the values of the charge density, $\rho_{0}$, and its three principal curvatures, $\rho_{\|\| \|}, \rho_{h h}$, and $\rho_{v v}$, respectively. The change in character of cps correlates with the energetics. For all three metals, $\rho_{h h}$ vanishes near the unstable stacking configuration. The symmetry or asymmetry of the charge redistribution, measured by $\rho_{h h} / \rho_{v v}$, may be an important factor determining stacking fault energies.
\end{abstract}

DOI: 10.1103/PhysRevLett.88.125501

PACS numbers: 61.72.Nn, 62.20.Fe, 71.15.Nc

The relationship between electronic structure and mechanical properties of materials has received considerable attention, with emphasis on developing atomistic models of fracture and deformation. Briant and Messmer [1] were among the first to use electronic structure calculations to investigate the effects of chemistry on the mechanical properties of metals and alloys. In the intervening years, considerable effort has been expended attempting to expand this work and provide an atomic level understanding of mechanical behavior [2-18]. This effort has been especially concerned with uncovering the electronic factors contributing to the competition between ductile and brittle response, as brittle failure frequently limits the use of alloys possessing otherwise attractive properties.

Over the past two decades, the tools of electronic structure theory have advanced tremendously. Now it is possible to investigate many of the structures giving rise to mechanical response (grain boundaries, planar faults, dislocations, etc.) and to compute the ground-state and thermodynamic properties associated with these structures [2-18]. Though the energies of point, line, and planar defects can now be computed, in some cases more accurately than they can be measured, it has proven difficult to assign these energy changes to specific changes in the charge density. This is because the charge density is a scalar field and changes in energy associated with a defect or mechanical perturbation are necessarily the result of changes to the whole field. We have sought to circumvent this problem by seeking a correlation between changes in the total energy and changes of the charge density in the neighborhood of a finite number of points, namely the critical points (cps) of the charge density.

In this work we employ ab initio calculations based on the full potential linear muffin tin orbital method [6], to study the energetics and the evolution of the electronic charge density with shear in three fcc metals, aluminum, silver, and iridium, exhibiting different deformation properties. The first two are prototypical fcc systems and as pure metals do not undergo cleavage fracture. These metals have different intrinsic stacking fault energies, $\gamma_{i s}$; $\gamma_{i s}=0.166 \mathrm{~J} / \mathrm{m}^{2}$ in $\mathrm{Al}$, about 6 times larger than that of $0.030 \mathrm{~J} / \mathrm{m}^{2}$ in $\mathrm{Ag}$ [19]. On the other hand, iridium is an anomalous fcc metal occasionally showing cleavage facets on its failure surface, and is characterized by a large intrinsic stacking fault energy of $0.400 \mathrm{~J} / \mathrm{m}^{2}$ [19].

Our analysis of charge redistribution is built on Bader's theory of atoms in molecules [20,21], where a relation between the molecular and solid state structures and the topological properties of the corresponding charge density was established. The topology of any scalar field is given in terms of its critical points, which are the zeros of the gradient of this field. There are four kinds of cps in a threedimensional space: a local minimum, a local maximum, and two kinds of saddle points. These cps are denoted by the number of positive curvatures minus the number of negative curvatures. For example, a minimum cp has positive curvature in three orthogonal directions. It is called a $(3,3) \mathrm{cp}$, where the first number is simply the number of dimensions of the space, and the second number is the net number of positive curvatures. A maximum is denoted by $(3,-3)$, since all three curvatures are negative. A saddle point with two of the three curvatures negative is denoted $(3,-1)$, while the other saddle point is a $(3,1) \mathrm{cp}$. Bader realized that the classification of molecular charge density in terms of the positions of its cps could be used to describe much of what is considered chemical structure and bonding $[20,21]$. The bond path, as an example, is seen as the ridge of maximum charge density connecting two nuclei. The existence of such a ridge is guaranteed by the presence of a $(3,-1) \mathrm{cp}$ between the bound nuclei. This $\mathrm{cp}$ is referred to as a bond critical point (bcp). Other types of critical points have been correlated with other features of molecular structure. A $(3,1) \mathrm{cp}$ is required at the center of ring structures like benzene and is referred to as a ring critical point (rcp). Cage structures are always characterized by a single $(3,3) \mathrm{cp}$ somewhere within the cage and again have 
been given the descriptive name cage critical points (ccp). The cps can be classified into those of rank three which are nondegenerate and stable and those of rank less than three which are degenerate and inherently unstable. The different types of structural changes that accompany the formation of an unstable $\mathrm{cp}$ can be classified in terms of its co-rank (number of zero curvatures) and the associated codimension [20,21]. We assume that the energy changes associated with shear are dominated by the breaking and forming of bonds, which must necessarily be accompanied by the vanishing and appearance of bcps. These in turn can be ascertained by following the charge density and its three principal curvatures at the cps through the distortion.

The shear process is produced by the relative translation of two parts of a crystal through a fault vector in the $\langle 112\rangle$ direction $[17,18]$. We have used a supercell containing nine layers in the [111] direction, multiple sets of energy parameters, and a $k$-point grid of $(15,15,3)$ divisions along the reciprocal lattice directions [6]. The three frames in Fig. 1 show the configurations corresponding to (a) the fcc structure, (b) the unstable stacking fault, and (c) the intrinsic stacking fault, respectively. Figure 1 restricts attention to the breaking and forming interplanar bonds accompanying the shear. The location of the cps associated with these bonds is marked by circles in the figure. In Fig. 1a the circle on the left marks a bcp while that on the right marks a ccp, being located at the center of an octahedral hole. At the end point of the distortion (Fig. 1c), the situation is reversed. The charge density at these points is denoted by $\rho_{0}$, while the principal curvatures are denoted by $\rho_{\||\||}, \rho_{h h}$, and $\rho_{v v}$. Here $\|$ denotes the direction parallel to either the breaking or forming bond (note that this direction changes through the distortion), $h h$ is the $\langle 110\rangle$ direction (this is the out of page, hence "horizontal," direction in Figs. 1 and 3), and $v v$ is the direction normal to the plane formed by $h h$ and the parallel component of the forming or breaking bond (this direction lies in the page of Figs. 1 and 3 and has a "vertical" component.)

Figure 2 shows the calculated change in the total energy versus the shear displacement, normalized to the displacement $(a / \sqrt{6})$ of the intrinsic stacking fault configuration for $\mathrm{Al}, \mathrm{Ag}$, and Ir, respectively. Also shown as a function (a)

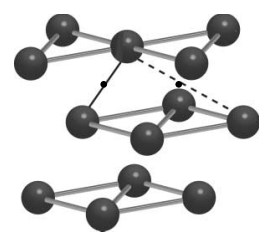

(b)

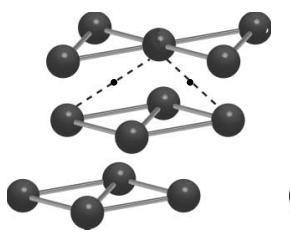

(c)

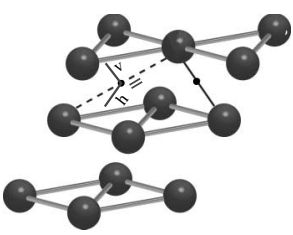

FIG. 1. Configurations corresponding to (a) the fcc structure, (b) the unstable stacking fault, and (c) the intrinsic stacking fault, for the (111) slip plane and shear deformation along the $\langle 112\rangle$ direction. The solid (dashed) lines denote the breaking (forming) interplanar bonds and the circles denote the corresponding critical points. The $h, v$, and $\|$ directions are shown at a cp in frame (c). of shear are the ab initio calculated $\rho_{0}, \rho_{\|\| \|}, \rho_{h h}$, and $\rho_{v v}$ at both the left (breaking bond) and right (forming bond) cps in Fig. 1. The solid (dashed) curves denote the breaking (forming) bonds. From this figure the nature of the charge redistribution associated with shear can be ascertained. First, we note that the redistribution of charge density can be characterized by three distinct regions. In the initial phase of the distortion, called "initiation," charge is lost from the bcp of the breaking bond more rapidly than it is being accommodated in the ccp of the forming bond. Inspection of the curvature panel of Fig. 2 shows that the charge redistribution is dominated by the rearrangement of charge around, rather than between, the transforming bond and cage cps. In the plane perpendicular to the forming bond, charge flows toward the $\mathrm{cp}$, causing the curvature in the perpendicular directions to decrease while that in the parallel direction increases. For the breaking bonds, the situation is reversed, with charge flowing away from the bcp in the plane perpendicular to the bond. The initiation region continues until the charge density in the horizontal direction, i.e., perpendicular to the direction of slip and parallel to the slip plane, becomes flat $\left(\rho_{h h} \rightarrow 0\right)$. This marks the onset of charge transfer between the forming and breaking bcps. We call this the "compensatory charge redistribution" region. In all cases this region is bounded on the left by the vanishing of $\rho_{h h}$ in the breaking bond and on the right by its vanishing in the forming bond.

The vanishing of $\rho_{h h}$ at the bond and cage cps necessitates the simultaneous vanishing of ring cps joined to them by gradient paths lying along the $h$ direction. Hence the region of compensatory charge redistribution is marked by a global flattening of the charge density along the $h$ direction and passing through vanishing bond, ring, and cage cps. The vanishing of the curvature in the $h$ direction produces planes normal to the shear in which the Gaussian curvature of the charge density vanishes. Figure 3 shows the charge density in a plane perpendicular to the forming bond near the region of compensatory charge redistribution. The flat charge density in the $h$ direction is clearly visible. It is in this second region of charge redistribution that the unstable stacking configuration is encountered. It is important to note that in the case of $\mathrm{Ag}$ and Ir the charge density is also flat in the $v$ direction $\left(\rho_{v v} \rightarrow 0\right)$ in this region. On the other hand, in Al $\rho_{v v} \rightarrow 0$ very early (late) in the bond breaking (forming) processes. Finally, in the final "relaxation" region, charge is again redistributed perpendicular to the shear direction, with charge flowing toward the forming bcp from forming rcps and away from the vanishing bcp into rcps in the planes perpendicular to the forming and breaking bonds. Consistent with the propagation of a distortion through a rigid body, it appears that a necessary condition for the propagation of a shear may be the vanishing of the Gaussian curvature in planes perpendicular to the shear.

Among the factors that influence the relative energies of the unstable stacking configurations is the shear required 

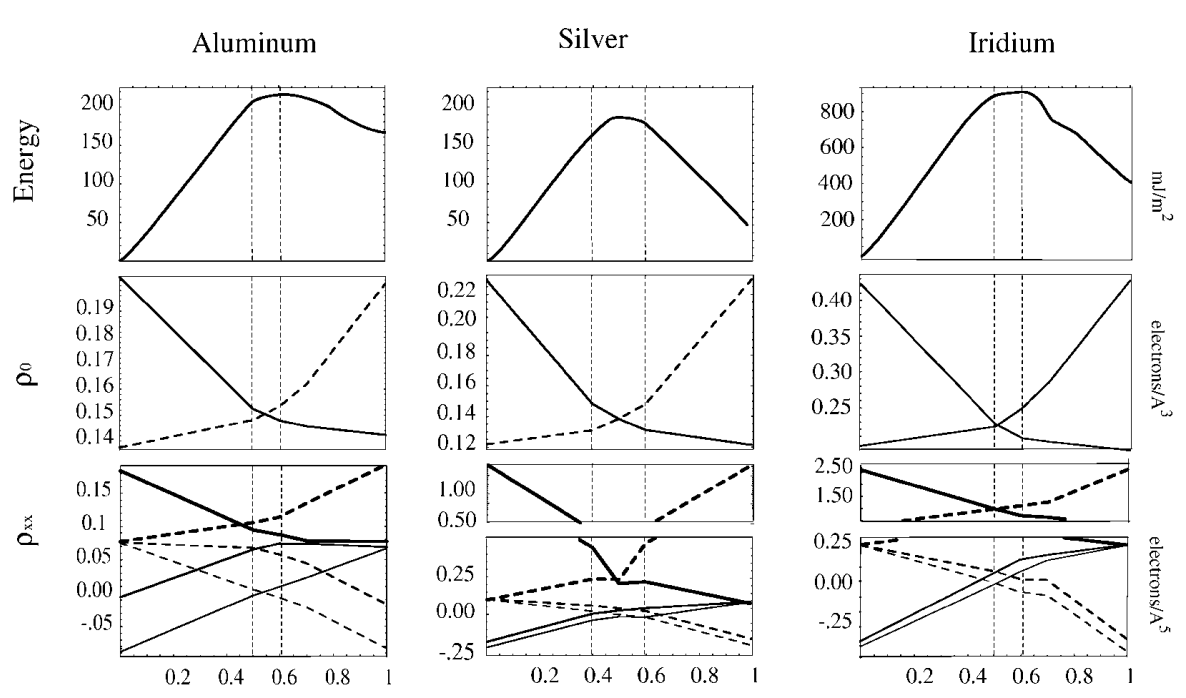

$$
\begin{array}{lll}
\rho_{\mathrm{III}} \text { Breaking }- & \rho_{\mathrm{v}} \text { Breaking }- & \rho_{\mathrm{hh}} \text { Breaking }- \\
\rho_{\mathrm{III}} \text { Forming - - } & \rho_{\mathrm{vy}} \text { Forming - - } & \rho_{\mathrm{hh} \text { Forming }---}
\end{array}
$$

before the onset of the compensatory charge redistribution. In $\mathrm{Ag}$, this type of charge redistribution begins $40 \%$ of the way through the shear, while for $\mathrm{Al}$ and Ir it begins at 50\%. The energy versus shear graphs for $\mathrm{Al}$ and $\mathrm{Ag}$ demonstrates that it is only because the region of compensatory charge redistribution occurs later for aluminum metal that it possesses a higher unstable stacking energy than does silver.

At the end point of the relaxation region, the charge density of the stacking fault is established. Notably, for all three metals, the charge density around the changing bond and cage cps of the stacking fault is nearly indistinguishable from that of the initial crystal. The values of $\rho_{0}, \rho_{\|\| \|}$, $\rho_{h h}$, and $\rho_{v v}$ for both the left and right hand critical points of Fig. 2 have simply been reversed. The initial and final values of these parameters then give no insight as to the magnitude of $\gamma_{i s}$. However, the nature of the charge redistribution does correlate with the energetics of the distortion. By way of example, the redistribution of charge in the case of Ag is seen to be nearly symmetric around the midpoint of the distortion. The initiation and relaxation regions appear similar. This same symmetry is reflected in the energy versus distortion curve of Ag. The charge redistribution of $\mathrm{Al}$ is distinctly asymmetric, with the values of $\rho_{\|\| \|}$ and $\rho_{v v}$ for the breaking bond behaving very differently from their counterparts in the forming bond. This asymmetry is reflected in the energetics of the Al distortion, reflecting the higher stacking fault energy of $\mathrm{Al}$ than $\mathrm{Ag}$.

We conjecture that the symmetry or asymmetry of the charge redistribution results from the way in which charge is partitioned between the tetrahedral and octahedral holes of fcc crystals. This partitioning is manifested by the comparative values of $\rho_{h h}$ and $\rho_{v v}$ at the bcp of the undistorted crystal. When $\rho_{h h}<\rho_{v v}$, as in Al, the average charge density in the tetrahedral hole exceeds that in the octahedral hole. On the other hand, as in Ag where $\rho_{h h} \approx \rho_{v v}$ the average density in the two holes is comparable. For the vanishing bcp at the beginning of the distortion there is no symmetry restriction on the comparative values of $\rho_{\|\| \|}$, $\rho_{h h}$, and $\rho_{v v}$. However, at the end of the distortion, in the intrinsic stacking fault configuration, the first coordination sphere around what has now become a ccp is octahedral. It is only the second coordination sphere that lowers this symmetry to $D_{3 h}$. At the ccp of this nearly octahedral field, symmetry requires that $\rho_{\|\| \|} \approx \rho_{h h} \approx \rho_{v v}$. This symmetry restriction is seen in all three metals through the computed values of principal curvatures for the breaking bcp. For the forming bond, the situation is reversed.

During the initiation phase, it appears that there is no preferred direction to the charge redistribution at the vanishing bcp. Charge flows from the parallel direction, uniformly in the horizontal and vertical directions, as indicated by the nearly parallel traces of the breaking bond $\rho_{h h}$ and $\rho_{v v}$ versus distortion (Fig. 2). Because $\rho_{v v}>\rho_{h h}$ for Al, $\rho_{v v}$ becomes comparable to $\rho_{\|\| \mid}$before $\rho_{h h}$ does. When $\rho_{\|\| \|} \approx \rho_{v v}$, these two quantities are effectively "pinned" by symmetry and further charge redistribution is now limited to flows that change the value of $\rho_{h h}$ only. Thus, charge redistribution is now constrained, accounting for its asymmetry in Al. The constrained charge redistribution in the relaxation phase manifests itself energetically as a reduction in the stabilization energy.

Our conjecture suggests that charge density anisotropy is a factor in determining the stacking fault energy of fcc metals. Those metals (Al) in which charge is preferentially partitioned in the tetrahedral hole will have higher values of $\gamma_{i s}$ than where the density is more equitably partitioned. The greater charge density, found in the tetrahedral holes of $\mathrm{Al}$, derive from wave functions near the Fermi energy that direct charge toward these holes [22]. Alloying elements that lower the Fermi energy will result in a more uniform charge density, and should lower the stacking fault energy of Al. Thus, metals in the same row of the periodic table and to its left, i.e., magnesium, should lower $\gamma_{i s}$, as is observed. 

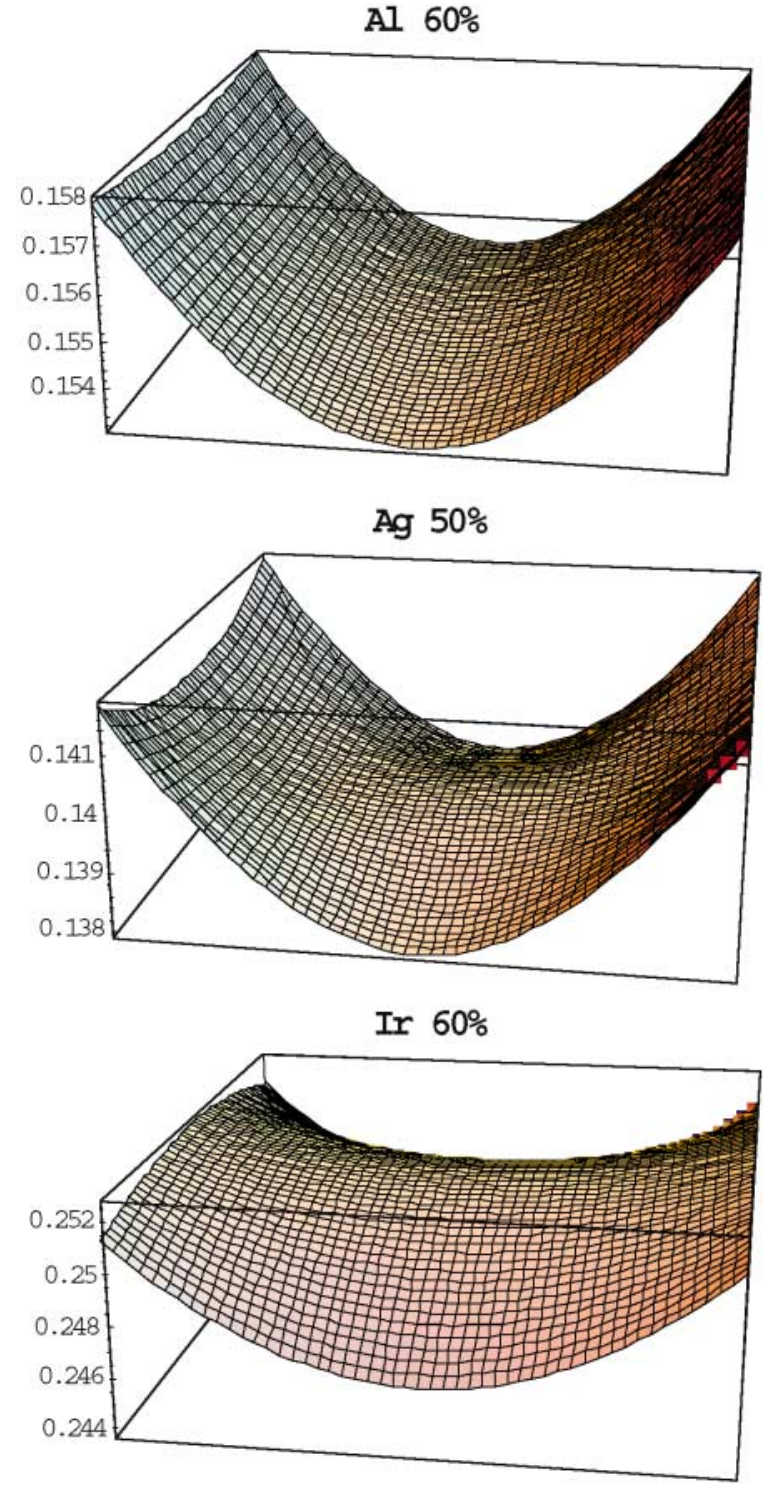

FIG. 3 (color). Charge density in a plane perpendicular to the forming bond containing the critical point near the region of "compensatory charge" redistribution for aluminum, silver, and iridium, respectively.

Extending our conjecture, interstitial alloying elements, which preferentially direct charge through $p$ or $d$ orbitals along one direction, should promote slip in the plane containing this direction. This is inferred from the observation that $\rho_{h h}$ must vanish just before the unstable stacking configuration is achieved. Charge from a directed $p_{z}$ orbital possess a node and thus contribute no curvature in the plane normal to the directed orbital. Such alloying elements will cause the charge density to become flatter in the nodal plane, and in response $\rho_{h h}$ will vanish earlier in the distortion than in the absence of the alloying element. Consequently, the unstable stacking configuration will occur at lower energies.

In summary, we have employed ab initio calculations to study the energetics and the evolution of the charge density with shear in three fcc metals, Al, Ag, and Ir, exhibiting different deformation properties. The charge redistribution accompanying this process is described by the change in character of specific charge density cps, with bond breaking associated with a transformation of a bcp to ccp, while the bond formation is associated with the reverse. For all three materials, $\rho_{h h}$ vanishes near the unstable stacking fault configuration. We find that the symmetry or asymmetry of the charge redistribution, as measured by $\rho_{h h} / \rho_{v v}$, may be an important factor determining the stacking fault energies in fcc metals.

The research at California State University was supported through the U.S. Army under Grants No. DAAG5597-1-0093 and No. DAAD19-00-1-0049. M.E.E. is grateful to the Air Force Office of Scientific Research, the DARPA, and the Petroleum Research Fund of the ACS for their support of this research.

[1] R.P. Messmer and C. L. Briant, Acta Metall. 30, 457 (1982).

[2] M. E. Eberhart, Science 265, 332 (1994).

[3] M. E. Eberhart and D. D. Vvedensky, Phys. Rev. B 37, 8488 (1988).

[4] P. A. Schultz and James W. Davenport, J. Alloys Compd. 197, 229-242 (1993).

[5] C. L. Fu and G. S. Painter, J. Mater. Res. 6, 719 (1991).

[6] S. N. Sun, N. Kioussis, and M. Ciftan, Phys. Rev. B 54, 3074 (1996).

[7] M.E. Eberhart and D. D. Vvedensky, Phys. Rev. Lett. 60, 1981 (1988).

[8] G. S. Painter and F. W. Averill, Phys. Rev. Lett. 58, 234 (1987).

[9] D. D. Vvedensky and M.E. Eberhart, Philos. Mag. Lett. 55, 157 (1987).

[10] M. E. Eberhart and D. D. Vvedensky, Scr. Metall. 22, 1183 (1988).

[11] S. Crampin et al., Phys. Rev. B 40, 3413 (1989).

[12] G. Lu, N. Kioussis, R. Wu, and M. Ciftan, Phys. Rev. B 59, 891 (1999).

[13] R. Wu, A.J. Freeman, and G. B. Olson, Science 265, 376-380 (1994).

[14] T. Hong and A. J. Freeman, Phys. Rev. B 43, 6446 (1991).

[15] C. Woodward, J. M. MacLaren, and S. Rao, J. Mater. Res. 7, 1735 (1992).

[16] J. M. MacLaren, S. Crampin, D. D. Vvedensky, and M. E. Eberhart, Phys. Rev. Lett. 63, 2586 (1989).

[17] G. Lu, N. Kioussis, V. Bulatov, and E. Kaxiras, Phys. Rev. B 62, 3099 (2000).

[18] G. Lu, Q. Zhang, N. Kioussis, and E. Kaxiras, Phys. Rev. Lett. 87, 095501 (2001).

[19] L. E. Murr, Interfacial Phenomena in Metals and Alloys (Addison-Wesley, Reading, MA, 1975).

[20] R. F. W. Bader, T. T. Nguyen-Dang, and Y. Tal, Rep. Prog. Phys. 44, 893 (1981).

[21] Richard F.W. Bader, Atoms in Molecules: A Quantum Theory (Oxford University Press, New York, 1990).

[22] M. E. Eberhart, Phys. Rev. Lett. 87, 205503 (2001). 